\title{
A study of mefloquine treatment for progressive multifocal leukoencephalopathy: results and exploration of predictors of PML outcomes
}

\author{
David B. Clifford • Avindra Nath • Paola Cinque • \\ Bruce J. Brew • Robert Zivadinov • Leonid Gorelik • \\ Zhenming Zhao $\cdot$ Petra Duda
}

Received: 23 March 2013 /Revised: 3 May 2013 / Accepted: 7 May 2013 / Published online: 4 June 2013

(C) The Author(s) 2013. This article is published with open access at Springerlink.com

\begin{abstract}
Immune reconstitution has improved outcomes for progressive multifocal leukoencephalopathy (PML), a potentially lethal brain disease caused by JC virus (JCV). However, an antiviral treatment to control JCV is needed when immune reconstitution is delayed or not possible. On the basis of in vitro efficacy, this study evaluated the effect of mefloquine on PML and factors that may predict PML outcomes. This 38-week, open-label, randomized, parallelgroup, proof-of-concept study compared patients with PML who received standard of care (SOC) with those who received SOC plus mefloquine (250 mg for 3 days, then
\end{abstract}

Electronic supplementary material The online version of this article (doi:10.1007/s13365-013-0173-y) contains supplementary material, which is available to authorized users.

D. B. Clifford $(\bowtie)$

Department of Neurology, Washington University

in St. Louis, Box 8111, 660 South Euclid Avenue,

St. Louis, MO 63110, USA

e-mail: cliffordd@neuro.wustl.edu

\section{A. Nath}

Section of Infections of the Nervous System,

National Institute of Neurological Disorders and Stroke,

National Institutes of Health, Bldg 10/ 7C-103, 10 Center Drive,

Bethesda, MD 20892, USA

P. Cinque

Department of Infectious Diseases, San Raffaele Scientific Institute, Via Stamira d'Ancona 20,

20127, Milan, Italy

\section{B. J. Brew}

Departments of Neurology and HIV Medicine, St Vincent's Hospital and University of New South Wales, Sydney, Australia

R. Zivadinov

Department of Neurology, Buffalo Neuroimaging Analysis Center, Buffalo, NY, USA
$250 \mathrm{mg}$ weekly). Patients randomized to SOC could add mefloquine treatment at week 4 . The primary endpoint was change from baseline to weeks 4 and 8 in JCV DNA copy number (load) in cerebrospinal fluid (CSF). Exploratory analyses evaluated factors that might correlate with clinical outcome. The majority of enrolled patients were HIV positive. Preplanned interim data analyses suggested that the study was unlikely to successfully demonstrate a significant difference between groups; therefore, the study was terminated prematurely. There was no significant difference between groups in CSF JCV DNA loads or clinical/MRI

\section{Gorelik}

Department of Research, Biogen Idec Inc., Weston, MA, USA

\section{Z. Zhao}

Pharmaceutical Product Development, LLC,

7901 E. Riverside Drive,

Austin, TX 78744, USA

P. Duda

Department of Medical Research, Biogen Idec Inc.,

Weston, MA, USA

\section{B. J. Brew}

Department of Neurology, Level 4, Xavier Building, St. Vincent's Hospital, Victoria Street Darlinghurst,

20120, Sydney, Australia

\section{R. Zivadinov}

Jacobs Neurological Institute, 100 High Street,

Buffalo, NY 14203, USA

L. Gorelik $\cdot$ P. Duda

Biogen Idec Inc., 14 Cambridge Center,

Cambridge, MA 02142, USA 
findings. Decrease in CSF JCV DNA load from baseline to week 4 was associated with a better clinical outcome at 16 weeks, as measured by Karnofsky scores. This study found no evidence of anti-JCV activity by mefloquine. An early decrease of CSF JCV DNA load appears to be associated with a better clinical outcome.

Keywords Mefloquine $\cdot$ Leukoencephalopathy $\cdot$ Progressive multifocal $\cdot \mathrm{JC}$ virus $\cdot$ Magnetic resonance imaging

\section{Introduction}

Progressive multifocal leukoencephalopathy (PML) is a serious brain infection caused by lytic JC virus (JCV) infection of oligodendrocytes and astrocytes in the human brain (Tan and Koralnik 2010; Brew et al. 2010; Ferenczy et al. 2012). PML occurs primarily in the setting of immune dysfunction, most commonly in untreated patients with advanced human immunodeficiency virus (HIV) infections, but also in the setting of malignancies, organ transplantation, and autoimmune diseases treated with certain immunomodulatory agents, including natalizumab. In untreated HIV patients, PML generally is rapidly fatal within months of diagnosis (Koralnik 2006).

With successful immune reconstitution, such as with highly active antiretroviral therapy (HAART) for patients with HIV infection, the prognosis for patients with PML has substantially improved (Berger 2011). However, direct antiviral therapy is needed for situations where immune reconstitution is difficult to achieve or delayed. Additionally, JC viral control entirely by immune reconstitution carries with it risk of "bystander" brain damage from excess immune responses that might be minimized by use of antiviral therapy. Thus far, there has been no such antiviral agent with proven efficacy despite anecdotal reports regarding various agents (Brew et al. 2010).

Mefloquine is a drug that has been used extensively for prophylaxis and treatment of malaria (Roche Laboratories Inc. 2003). Previously, an in vitro model of JCV infection of human astrocytes was used to screen several thousand available drugs for activity against JCV. Mefloquine was found to inhibit JCV infection and replication in concentrations generally achieved in brains of patients given mefloquine for malaria (Brickelmaier et al. 2009). This activity included the ability to inhibit infection with JCV strains typically causing PML.

Since there is no animal model available with which to study PML effectively (Brickelmaier et al. 2009) and mefloquine has a long and substantial history of use and generally favorable record of safety in humans (Schlagenhauf et al. 2010), it was decided to undertake a clinical trial seeking evidence of clinical activity of mefloquine in patients with PML, without further in vitro or animal studies. The clinical trial also provided a rare opportunity to systematically study factors that may be predictive of patient outcomes in PML, while providing guidance for the design for future PML clinical studies.

\section{Methods}

Study design

An open-label, rater-blinded, randomized, parallel-group treatment trial comparing a group receiving only local standard-of-care (SOC) therapy for PML (which could include any treatment or procedure that the investigator would normally use to treat PML; hereafter referred to as "SOC" or "control" group) with a group receiving both SOC and mefloquine ( $250 \mathrm{mg}$ daily for 3 days, then weekly; hereafter referred to as "mefloquine group") was undertaken (Fig. 1). The primary endpoint was the change from baseline to weeks 4 and 8, respectively, in JCV DNA load in the cerebrospinal fluid (CSF).

The SOC group was given the option to receive mefloquine treatment beginning at week 4 (or week 8 in early versions of the protocol). The planned duration of the study, including screening, 24 weeks of treatment, and follow-up, was 38 weeks. Patients with PML were recruited at 13 study sites [US (five sites), Germany (three sites), Spain (two sites), Italy (one site), Australia (one site), and Brazil (one site)]. The study protocol and consent forms were approved by the institutional review boards/ethics committees associated with the study sites. All patients gave informed consent for this study.
Fig. 1 Trial design. Asterisk In an earlier version of the protocol, patients could cross over to SOC + mefloquine at week 8 . The final protocol allowed this option at week 4. $M$ month, $P M L$ progressive multifocal leukoencephalopathy, $S O C$ standard of care






\section{Patients}

Eligible patients were men or women with PML, between 18 and 75 years of age. Other eligibility criteria included weight $\geq 30 \mathrm{~kg}$, confirmation of PML diagnosis by JCV DNA detection in CSF, onset of PML symptoms within 6 months of randomization, and anticipated survival of $\geq 2$ months. Concomitant opportunistic central nervous system infections or unexplained epileptic seizures within 6 months pre-randomization were exclusionary. Both HIV-positive and HIV-negative patients were included. HIV-positive patients received antiretroviral therapy as SOC.

\section{Assessments}

JCV DNA copy number in the CSF was measured at screening and at 4, 8, 16, and 24 weeks at a central laboratory, Focus Diagnostics, Inc. (Cypress, CA, USA). The lower limit of quantitation was 50 copies $/ \mathrm{mL}$. Other laboratory values including $\mathrm{CD}^{+} / \mathrm{CD}^{+}$fluorescence-activated cell sorter (FACS) count, $\mathrm{CD}^{+} / \mathrm{CD}^{+}$FACS count, Cylex ${ }^{\circledR}$ (Cylex Inc., Columbia, MD, USA) stimulated adenosine triphosphate (ATP), and HIV RNA polymerase chain reaction (PCR) were evaluated at screening and at every regularly scheduled study visit. Serum mefloquine levels were measured throughout the treatment period; mean steadystate levels were modeled relative to JCV DNA levels in the CSF. Plasma concentrations of mefloquine and carboxymefloquine, determined by mass spectroscopy (mefloquine assay and carboxylic acid assay), were measured at baseline and at weeks 4, 8, 16, and 24. Brain mefloquine concentration in the frontal lobe was measured in the case of one patient who died 2 days after mefloquine dosing.

Clinical efficacy measures included the Karnofsky Performance Scale Index (KPSI), Expanded Disability Status Scale (EDSS), and the Symbol Digit Modalities Test (SDMT) at baseline and at 4, 8, 16, and 24 weeks. A Visual Analogue Scale (VAS), with which the patient indicated his/her impression of overall well-being, was included at screening and at every regularly scheduled visit.

Brain MRI scans were performed at baseline and at 4, 8, 16, and 24 weeks, and image analyses were at the Buffalo Neuroimaging Analysis Center, University of Buffalo, New York. MRI sequences included 2D multiplanar dual fast spin-echo proton density and T2-weighted image (WI), fluid-attenuated inversion-recovery, and spin echo T1-WI both with and without a single-dose intravenous bolus of $0.1 \mathrm{mmol} / \mathrm{kg}$ gadolinium $(\mathrm{Gd})$-diethylenetriaminepentaacetic acid with scanning $5 \mathrm{~min}$ after injection. The "Electronic Supplementary Material" gives a more detailed description of MRI sequences.
$\mathrm{T} 2$, $\mathrm{T} 1$, and gadolinium-enhancing $(\mathrm{Gd}+)$ lesion volumes (LVs) in areas suggestive of PML were calculated using a semi-automated edge-detection contouringthresholding technique, as previously described (Zivadinov et al. 2001). Non-serious adverse events, serious adverse events, and concomitant therapy were monitored on an ongoing basis. An independent safety monitoring committee was appointed to provide safety oversight for the study. Mortality was assessed throughout the study.

\section{Statistical analysis}

The population for efficacy analysis consisted of all patients who had a measurable JCV DNA load in CSF at screening from the central laboratory. The patients were stratified into three groups: (1) HIV-positive, HAART-naïve patients ( $n=$ 24); (2) HIV-positive patients with a history of HAART ( $n=$ $5)$; and (3) HIV-negative patients $(n=8)$. Patients who switched to mefloquine treatment at week 4 or week 8 were counted in both comparison groups (SOC vs mefloquine). All patients who entered the study and had a postbaseline safety assessment were included in the safety analyses. The population for pharmacokinetic analysis consisted of all patients who received mefloquine and had at least one measurable mefloquine concentration. The study was powered to detect at least 1 log difference in JCV load between treatment groups, but not to detect differences in other measures.

Results of clinical and laboratory/imaging evaluations were expressed as mean values \pm standard deviation (SD), unless otherwise noted. The Student's $t$ test was used to analyze the change from baseline to week 4 (and week 8 in a sensitivity analysis) on the logtransformed JCV DNA level in CSF and MRI T2/T1 LVs as well. The Wilcoxon rank-sum test was used to analyze the changes in other endpoints including EDSS, KPSI, and SDMT. For patients who died, EDSS or KPSI scores at the time of death and up to week 24 were imputed to 10.0 or 0 , respectively (EDSS score of 10.0 or KPSI score of 0 signifies death). The Fisher exact test was used to analyze MRI Gd activity and mortality.

As there was no difference detected between the mefloquine and SOC control groups on any measure, data were pooled for post hoc analyses to explore factors that may predict clinical outcome in patients with PML. Only data from HIV-positive patients were used for these exploratory analyses. The last observation carried forward (LOCF) method was used to impute the missing values for KPSI, and its change from baseline to week 16 was calculated. A categorical form of KPSI was then defined as improvement (increase in 
score $>1$ ), deterioration (decrease in score $>1$ ), or stable (patients who did not meet improvement or deterioration criteria).

The following variables of interest were tested for their potential correlation or association to KPSI change from baseline to week 16; except where specified, both baseline and change from baseline at week 4 results were tested: KPSI score ( $<50$ vs $\geq 50$, baseline only), EDSS score ( $<6.0$ vs $\geq 6.0$, baseline only), $\log _{10} \mathrm{JCV}$ DNA load in CSF, $\log _{10}$ JCV DNA load in plasma, $\log _{10} \mathrm{JCV}$ DNA load in urine, $\mathrm{CD}^{+} / \mathrm{CD}^{+}$FACS count, $\mathrm{CD}^{+} / \mathrm{CD}^{+}$FACS count, Cylex ${ }^{\circledR}$ stimulated ATP, HIV RNA PCR, presence of $\mathrm{Gd}+$ lesions, T2 volume, SDMT, and VAS. The Pearson correlation coefficient was calculated to evaluate relationships between variables. Logistic regression was used to explore the association between KPSI score and the variables of interest. The Fisher exact test was performed if there were issues of separation for binary variables, such as EDSS score $<6.0$ vs $\geq 6.0$ at baseline.

\section{Results}

\section{Patients}

Patient characteristics are presented in Table 1. Thirty-seven patients were randomized (29 HIV-positive and 8 HIV- negative patients) to SOC or mefloquine. Only 12 of the 37 patients who were randomized and dosed completed study treatment.

The study was terminated prematurely upon recommendation by the Data Safety Committee as ongoing data monitoring allowed conditional power calculations that suggested a very small probability of the study demonstrating any difference in the primary endpoint in this small study population. Calculations suggested that even if the study could have been extended to allow for a much larger patient population, it remained unlikely that a difference could be demonstrated.

Twenty-two serious adverse events were reported, predominantly associated with progressing neurological disability or death. None was believed to be associated with administration of mefloquine. Deaths were disproportionately represented in the HIV-negative group (four deaths among eight randomized patients, $50 \%$ ) vs HIV-positive group (four deaths among 29 randomized patients, $14 \%$ ). Many of the deaths occurred early in the trial, making attribution of findings to assigned therapy problematic. Results of the mefloquine study and exploratory analyses reported hereafter are limited to results in HIV-positive patients (both HAART-naïve patients and those with a history of receiving HAART), as meaningful analysis of data from HIV-negative patients was limited by the small number of patients, as well as by the early and high mortality in this group.

Table 1 Patient characteristics

\begin{tabular}{|c|c|c|c|c|c|c|}
\hline & & $\begin{array}{l}\text { Randomized } \\
\text { to SOC alone }\end{array}$ & $\begin{array}{l}\text { Randomized to } \\
\text { SOC, then added } \\
\text { mefloquine at } \\
\text { week } 4\end{array}$ & $\begin{array}{l}\text { Randomized to } \\
\text { SOC, then added } \\
\text { mefloquine at } \\
\text { week } 8\end{array}$ & $\begin{array}{l}\text { Randomized to } \\
\text { mefloquine }+ \text { SOC }\end{array}$ & Total \\
\hline Number of patients dosed & & 7 & 5 & 5 & 20 & 37 \\
\hline \multirow[t]{3}{*}{ Disease history, $n(\%)$} & HAART naïve & $3(43)$ & $5(100)$ & $3(60)$ & $13(65)$ & $24(65)$ \\
\hline & History of HAART & $1(14)$ & 0 & $1(20)$ & $3(15)$ & $5(14)$ \\
\hline & HIV negative & $3(43)$ & 0 & $1(20)$ & $4(20)$ & $8(22)$ \\
\hline \multirow{3}{*}{$\begin{array}{l}\text { JCV DNA titer (in CSF) } \\
\text { at screening (copies } / \mathrm{mL}) \text {, } \\
n(\%)\end{array}$} & $\leq 50$ & 0 & 0 & 0 & $4(20)^{\mathrm{a}}$ & $4(11)^{\mathrm{a}}$ \\
\hline & $>50$ & $7(100)$ & $5(100)$ & $5(100)$ & $15(75)$ & $32(86)$ \\
\hline & Missing & 0 & 0 & 0 & $1(5)$ & $1(3)$ \\
\hline Gender male, $n(\%)$ & Male & $6(86)$ & $3(60)$ & $3(60)$ & $15(75)$ & $27(73)$ \\
\hline \multirow[t]{2}{*}{ Race, $n(\%)$} & $\begin{array}{l}\text { Black or African } \\
\text { American }\end{array}$ & $1(14)$ & $2(40)$ & $2(40)$ & $4(20)$ & $9(24)$ \\
\hline & White & $6(86)$ & $3(60)$ & $3(60)$ & $16(80)$ & $28(76)$ \\
\hline Age (years), mean (SD) & & $48.7(20.56)$ & $41.6(11.37)$ & $47.4(10.06)$ & $48.1(9.40)$ & $47.2(12.16)$ \\
\hline Weight $(\mathrm{kg})$, mean (SD) & & $71.31(14.83)$ & $60.12(12.75)$ & $75.54(22.88)$ & $65.71(15.52)$ & $67.34(16.16)$ \\
\hline
\end{tabular}

HAART highly active antiretroviral therapy, HIV human immunodeficiency virus, JCV JC virus, $S D$ standard deviation, $S O C$ standard of care

All patients $(n=7)$ in the "randomized to SOC alone" column discontinued study treatment; of the remaining patients, those who were not randomized to receive SOC + mefloquine at the start of the study opted to be treated with mefloquine at week 4 or week 8 . No patients remained on SOC alone throughout the study

${ }^{\text {a }}$ Patients enrolled based on local laboratory data; in some instances, the results were not replicated by the central laboratory 


\section{CSF JCV DNA}

Baseline CSF JCV DNA levels ( $\log _{10}$ copies per milliliter) were the same in the SOC $(n=13$; mean 3.7, SD 1.18) and the mefloquine groups ( $n=19$; mean 3.7, SD 1.24). Mean CSF JCV DNA values at each time point were similar for the SOC and the mefloquine groups (Fig. 2). Overall mean changes in JCV DNA in CSF viral loads did not differ significantly between mefloquine and control groups at week $4(\mathrm{SOC}=-0.46 \pm 0.60$ vs mefloquine $=$ $0.04 \pm 1.54 ; p=0.2972$ ).

Exploratory analyses revealed that reductions in JCV DNA load in CSF at week 4 were significantly associated with improvement in KPSI at week 16 (the clinical outcome measure for the exploratory analyses; Table 2). This association was maintained at weeks 8 and 16 (Fig. 3). In contrast, JCV DNA loads in CSF at baseline and changes at week 4 did not correlate with KPSI values at other time points besides week 16.

Other clinical and laboratory variables

Mean KPSI scores at both weeks 4 and 8 were also (unexpectedly) higher in the SOC group (Fig. 4). Mean change in KPSI scores was significantly higher in the SOC group than in the mefloquine group at week $4(\mathrm{SOC}=5.56 \pm$ 7.27 vs mefloquine $=-5.56 \pm 11.50 ; p=0.0050$ ); however, this difference was not sustained to week 8 ( $\mathrm{SOC}=7.5 \pm$ 12.58 vs mefloquine $=-6.4 \pm 16.46 ; p=0.1190$ ). Also, the study was not powered to detect differences between treatment groups in other measures besides difference in JCV load.

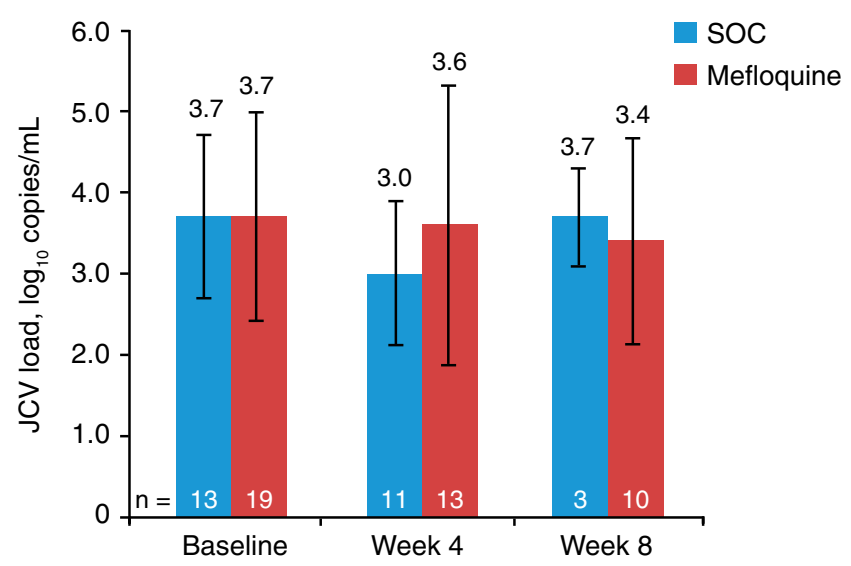

Fig. 2 Mean $( \pm$ SD) JCV DNA load in CSF in all HIV-positive patients (HAART-naïve and history of HAART groups combined). HIV-negative patients not included in analysis. Patients in the SOC group who opted to receive mefloquine at week 4 were not counted in the week 8 results. CSF cerebrospinal fluid, HAART highly active antiretroviral therapy, $H I V$ human immunodeficiency virus, JCV JC virus, $S O C$ standard of care
Mean EDSS scores were higher in the SOC group than in the mefloquine group (Fig. 5). Mean changes in EDSS score from baseline were not significantly different between mefloquine and control groups at week $4(\mathrm{SOC}=0.39 \pm 0.74 \mathrm{vs}$ mefloquine $=0.31 \pm 1.02 ; p=0.6190)$ or week 8 (SOC $=$ $-0.63 \pm 0.95$ vs mefloquine $=0.64 \pm 1.23 ; p=0.0588$ ).

Although the sample size was small and diminished over time ( $N=24$ at baseline and $N=14$ by week 15 ; weeks 20 and 24 not included in analyses), EDSS and KPSI values were correlated at baseline and at weeks 4 , 8, and 16 (Pearson correlation coefficient $[r]$ 0.768, $0.719,0.792$, and 0.858 , respectively) (Supplementary Figure 1). Compared with their actual values, KPSI and EDSS changes from baseline were not as highly correlated at weeks 4,8 , and 16 (data not shown).

Other clinical or laboratory variables at baseline or the change in their value from baseline to week 4 did not show any notable correlation to the clinical outcome measure (Table 2). Sensitivity binary analyses done by dividing the clinical outcome measure into those improving, remaining stable, or deteriorating on KPSI at week 16 did not reveal any additional information.

\section{Brain MRI findings}

There were no significant differences in MRI findings between mefloquine-treated patients and control patients either at baseline or following randomization. MRI showed $\mathrm{Gd}+$ lesions at baseline in eight of $18(44 \%)$ patients who received mefloquine and in six of 11 (55\%) control patients; at week 4, in eight of 12 (67\%) patients who received mefloquine and in five of ten $(50 \%)$ control patients; at week 8 , and in seven of $11(64 \%)$ patients who received mefloquine and in one of four $(25 \%)$ control patients. There were no significant differences in the change from baseline to subsequent time points in $\mathrm{T} 1, \mathrm{~T} 2$, or $\mathrm{Gd}+\mathrm{LV}$ s between mefloquine and control groups. There was no significant relationship between MRI parameters and the clinical outcome measure (Table 2).

\section{Discussion}

Mefloquine did not show evidence of in vivo antiviral activity against JCV as evaluated by JCV DNA load in CSF. While the study was specifically designed to measure differences in JCV load between patients treated with mefloquine and patients treated with SOC, there also did not appear to be any mefloquine effect on clinical disability, MRI parameters, or survival in comparison with SOC. Lack of efficacy likely was not because of inability to achieve therapeutic concentration 
Table 2 Explorations of potential predictors of clinical deterioration at week 16 as measured by change in KPSI to week 16

\begin{tabular}{|c|c|c|c|c|}
\hline \multirow[b]{2}{*}{ Variable of interest } & \multicolumn{2}{|c|}{ Baseline } & \multicolumn{2}{|c|}{ Change at week 4} \\
\hline & $N$ & OR $(95 \% \mathrm{CI})$ & $N$ & OR $(95 \% \mathrm{CI})$ \\
\hline $\mathrm{CD}^{+} / \mathrm{CD}^{+}{ }^{+}$FACS count $\left(\mathrm{mm}^{3}\right)$ & 14 & $0.999(0.993$ to 1.005$)$ & 10 & $0.983(0.949$ to 1.017$)$ \\
\hline $\mathrm{CD}^{+} / \mathrm{CD}^{+}$FACS count $\left(\mathrm{mm}^{3}\right)$ & 14 & $1.002(0.998$ to 1.005$)$ & 10 & $1.006(0.997$ to 1.016$)$ \\
\hline Cylex stimulated ATP (ng/mL) & 12 & $0.989(0.978$ to 1.001$)$ & 8 & $1.002(0.985$ to 1.020$)$ \\
\hline EDSS score $(<6, \geq 6)$ & 14 & $2.715(0.363$ to 20.292$)$ & 14 & $\mathrm{~N} / \mathrm{A}$ \\
\hline HIV RNA PCR (copies/mL) & 10 & $1.000(1.000$ to 1.000$)$ & 6 & $1.000(0.999$ to 1.002$)$ \\
\hline KPSI score $(<50, \geq 50)$ & 14 & $3.000(0.392$ to 22.933$)$ & 14 & $\mathrm{~N} / \mathrm{A}$ \\
\hline Presence of Gd + lesions & 12 & $5.000(0.520$ to 48.066$)$ & 9 & $0.291(0.016$ to 5.231$)$ \\
\hline SDMT score & 10 & $1.041(0.927$ to 1.169$)$ & 6 & $1.062(0.806$ to 1.398$)$ \\
\hline $\mathrm{T} 2$ lesion volume $\left(\mathrm{mm}^{3}\right)$ & 13 & $1.000(1.000$ to 1.000$)$ & 9 & $1.000(1.000 \text { to } 1.000)^{\mathrm{b}}$ \\
\hline VAS score & 13 & $1.003(0.974$ to 1.032$)$ & 10 & $1.015(0.984$ to 1.048$)$ \\
\hline $\log _{10}(\mathrm{JCV}$ DNA in CSF) (copies/mL) & 14 & $0.846(0.345$ to 2.074$)$ & 11 & $0.096(0.009 \text { to } 0.986)^{\mathrm{c}}$ \\
\hline $\log _{10}(\mathrm{JCV}$ DNA in plasma) (copies/mL) & 3 & $\mathrm{~N} / \mathrm{A}^{\mathrm{a}}$ & 0 & $\mathrm{~N} / \mathrm{A}$ \\
\hline $\log _{10}(\mathrm{JCV}$ DNA in urine) (copies/mL) & 8 & $1.513(0.567$ to 4.040$)$ & 5 & $1.494(0.237$ to 9.403$)$ \\
\hline
\end{tabular}

This table reflects ordinal logistic regression on variables of interest at baseline and change from baseline to week 4 . Odds ratios are given between patients who deteriorated on the KPSI score at week 16 compared with baseline vs those who did not. If no values were available at week 16 , those from week 12 were used. Decrease of JCV DNA load in CSF was the only variable significantly associated with lack of clinical deterioration $(p=0.015)$

$A T P$ adenosine triphosphate, $C I$ confidence interval, CSF cerebrospinal fluid, EDSS Expanded Disability Status Scale, FACS fluorescence-activated cell sorter, $G d+$ gadolinium enhancing, $H I V$ human immunodeficiency virus, JCV JC virus, KPSI Karnofsky Performance Scale Index, $L O C F$ last observation carried forward, $L R T$ likelihood ratio test, $N / A$ not applicable for testing under this condition (test did not provide any meaningful data), $O R$ odds ratio, $P C R$ polymerase chain reaction, SDMT Symbol Digit Modality Test, VAS Visual Analogue Scale

${ }^{\text {a }}$ Maximum likelihood modeling was unsuitable due to data separation issues

${ }^{\mathrm{b}}$ Before rounding to three digits after the decimal, the lower limit of the CI was slightly less than 1.000 and the upper limit of the CI was slightly greater than 1.000

${ }^{\mathrm{c}}$ For every unit increased in JCV load, the odds of improvement are 0.096 for no deterioration vs deterioration

of mefloquine; in one patient who received mefloquine 2 days prior to death, brain mefloquine concentration was measured at $84 \mu \mathrm{M}$ at autopsy.

It is important to note that only 12 of the 37 patients who were randomized and dosed completed study treatment; therefore, the data are limited. Because of the small number of patients with non-HIV-related PML included in this study and because of the early deaths in this arm, it was not possible to do a meaningful analysis of patients who were not HIV positive. The results therefore reflect PML in the setting of HIV disease.

The mortality rate for HIV-positive patients in the study was approximately $14 \%$, which is better than most historical reports (Gasnault et al. 2011) but also reflects a shorter time frame than do many reports. The rate also only reflects patients who completed the study; many patients did not finish the study period because of study termination or premature withdrawal and/or disease progression (thus, it is possible the mortality rate over a more extended period would be higher than $14 \%$ ). In light of apparent lack of mefloquine treatment effect on PML, the data were explored for information that might be associated with clinical outcomes in PML patients.
In the analysis of the association between the clinical outcome measure and various variables of interest, most were not significant. However, decreasing JCV DNA load in CSF as early as week 4 was correlated with better clinical outcome, consistent with earlier findings that demonstrated a marked decrease in JCV DNA levels over time in HAART-treated patients in whom the PML disease course stabilized (Bossolasco et al. 2005; Delbue et al. 2012).

Contrary to what might be expected, neither $\mathrm{CD} 4{ }^{+}$ cell counts nor serum HIV RNA titers at baseline were associated with the clinical outcome in this HIV-positive population. This likely reflects the availability of active HIV therapies capable of initiating immune reconstitution in most AIDS patients in the current era.

The small number of evaluable patients in this trial reflects the logistical and technical challenges inherent in PML clinical trials. While larger trials are needed to reach definitive conclusions, experience thus far indicates that it will be very challenging to enroll a sufficient number of PML patients in clinical trials. Also, the frequently rapid speed of PML disease progression may complicate attempts to monitor treatment effects over time in studies of this nature. Finally, the success of antiretroviral treatments, especially in the modern era, may 
Fig. 3 Correlation between KPSI change from baseline to 16 weeks and $\log 10$ JCV DNA load (in CSF) at baseline (a) and change to weeks 4,8 , and 16 (b-d) (with LOCF from week 12). CSF cerebrospinal fluid, JCV JC virus, KPSI Karnofsky Performance Scale Index, LOCF last observation carried forward
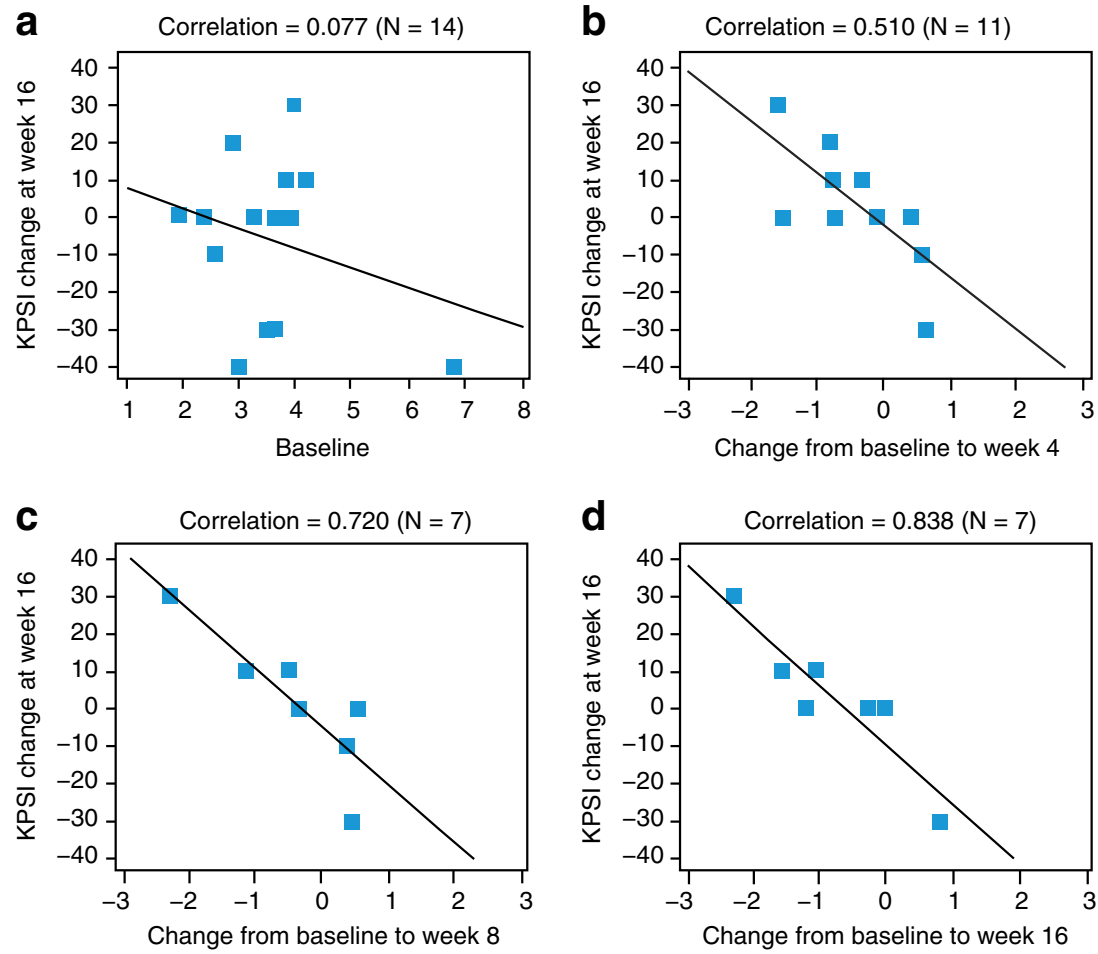

limit the power to demonstrate improved mortality in studies such as this one because it is likely to induce prompt immune reconstitution in most patients with PML.

This trial also underscores the specific challenges of recruiting for a randomized study that uses a commercially available drug. In the face of a potentially fatal disease, patients, families, and clinicians might be uncomfortable with a randomized trial and highly motivated to try the active treatment without demanding efficacy or toxicity data to support this decision.

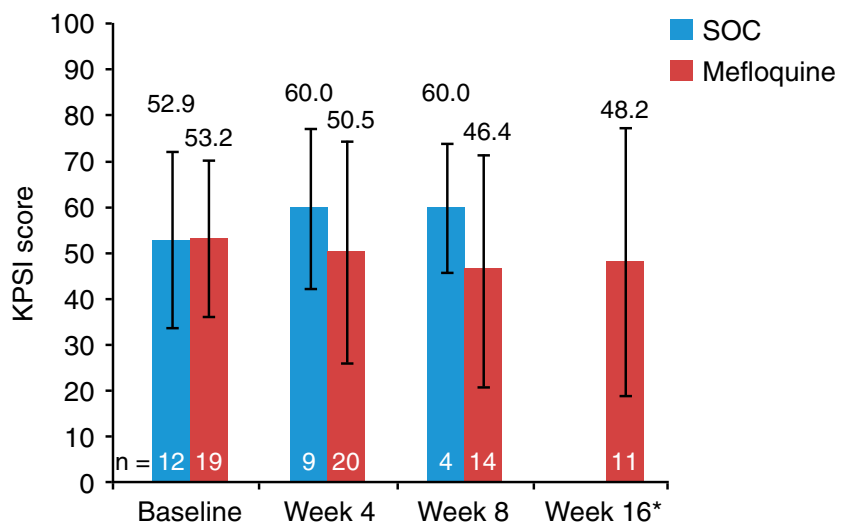

Fig. 4 Mean ( \pm SD) KPSI scores in all HIV-positive patients (HAART-naïve and history of HAART groups combined). HIV-negative patients not included in analysis. Patients on SOC who opted to receive mefloquine at week 4 were not counted in the week 8 results. Asterisk No data for SOC for this time point. HAART highly active antiretroviral therapy, HIV human immunodeficiency virus, KPSI Karnofsky Performance Scale Index, SOC standard of care
Our study is too small to definitively rule out mefloquine activity against PML, but given the inability to find any evidence of activity in this study, we would not advocate empiric use of this therapy for PML patients. The findings from our study support the use of change in JCV DNA in the $\mathrm{CSF}$, as early as 4 weeks into treatment, as a marker of clinical outcome in patients with PML. This may be helpful for management of PML patients, as well as in the design and conduct of potential future treatment trials in this population.

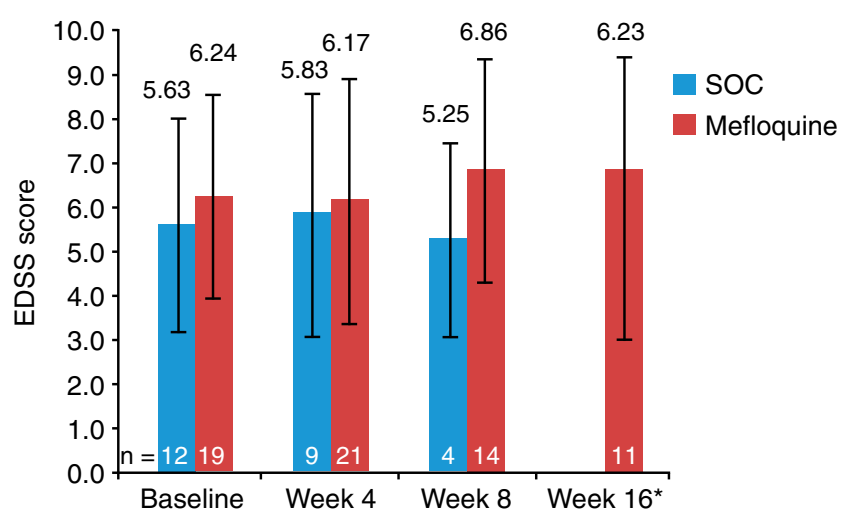

Fig. 5 Mean $( \pm \mathrm{SD})$ EDSS scores in all HIV-positive patients (HAART-naïve and history of HAART groups combined). HIV-negative patients not included in analysis. Patients who opted to receive mefloquine at week 4 were not counted in the week 8 results. Asterisk No data for SOC for this time point. EDSS Expanded Disability Status Scale, HAART highly active antiretroviral therapy, HIV human immunodeficiency virus, SOC standard of care 
Acknowledgments This study was supported by Biogen Idec Inc. and Elan Pharmaceuticals, Inc. Funding for assistance with medical writing, as well as editorial support, was also provided by Biogen Idec Inc. and Elan Pharmaceuticals, Inc. Exploratory analyses were performed by BioStat Solutions Inc., in collaboration with Nigel Dodd of Biogen Idec Inc. The first author, Dr. Clifford, prepared the initial draft; assistance in medical writing was provided by Katherine Hauswirth and editorial support was provided by Jackie Cannon, both of Infusion Communications. The authors would like to acknowledge the following principal investigators/sites that enrolled patients for this study: Keikawus Arasteh, MD, Auguste-ViktoriaKrankenhaus Klinikum, Berlin, Germany; Gabriele Arendt, MD, HeinrichHeine Universität, Neurologische Klinik, Düsseldorf, Germany; David B. Clifford, MD, Washington University in St. Louis, St. Louis, MO, USA; Bruce A. Cohen, MD, Northwestern University, Evanston, IL, USA; Igor Koralnik, MD, Beth Israel Deaconess Medical Center, Boston, MA, USA; Adriano Lazzarin, MD, Divisione di Malattie Infettive, Fondazione Centro San Raffaele del Monte Tabor, Milan, Italy; Justin McArthur, MBBS, MPH, Johns Hopkins University, Baltimore, MD, USA; Jose Miro, MD, Hospital Clinic I Provincial de Barcelona, Barcelona, Spain; Augusto César Penalva de Oliveira, Ph.D., Instituto de Infectologia Emillo Ribas, São Paulo, Brazil; Thorsten Rosenkranz, MD, Asklepios Klinik St. George, Hamburg, Germany; and David M. Simpson, MD, Mount Sinai Medical Center, New York, NY, USA. The authors would also like to acknowledge the investigators at the Buffalo Neuroimaging Analysis Center, Buffalo, NY, USA, who collected the scans and performed the analyses: Cheryl Kennedy, LMSW, MPH; Niels Bergsland, M.Sc.; Michael G. Dwyer, M.Sc.; Jackie Durfee, BS; Sara Hussein, BS; and Deepa P. Ramasamy, MD

Conflicts of interests D. C. serves on Data Safety Boards or PML Adjudication Committees for Amgen, Biogen Idec, Genentech, Genzyme, Millennium, and Pfizer. He has been a consultant to Biogen Idec, Bristol-Myers Squibb, Genentech, Genzyme, Janssen, Millennium, and Pfizer. He has received research support from Biogen Idec, NeurogesX, Pfizer, and Tibotec. He receives research support from the NIAID, NIMH, and NINDS. A. N has no conflict of interest. P. C. received personal compensation for consultancy, lectures, or board membership from Abbott, Astellas, Boehringer-Ingelheim, Biogen Idec, Bristol Myers-Squibb, Elan Pharmaceuticals, Gilead, Jannsen-Cilag, Johnson \& Johnson, Merck, Mundipharma, Pfizer, and Viiv Healthcare. She received financial support for research activity from Biogen Idec. B. J. B. received speaker honoraria from and was an advisory board member for Biogen Idec. R. Z. received personal compensation from Bayer, Biogen Idec, Bracco, EMD Serono, Genzyme-Sanofi, Novartis, Questcor Pharmaceuticals, and Teva Neuroscience for speaking and as a consultant. He received financial support for research activities from Biogen Idec, Bracco, EMD Serono, Genzyme-Sanofi, Novartis, Questcor Pharmaceuticals, and Teva Neuroscience. L. G. and P. D. are employees of Biogen Idec. Z. Z. is a former employee of Biogen Idec.

Open Access This article is distributed under the terms of the Creative Commons Attribution License which permits any use, distribution, and reproduction in any medium, provided the original author(s) and the source are credited.

\section{References}

Berger JR (2011) The clinical features of PML. Cleve Clin J Med 78(suppl 2):S8-S12

Bossolasco S, Calori G, Moretti F, Boschini A, Bertelli D, Mena M, Gerevini S, Bestetti A, Pedale R, Sala S, Sala S, Lazzarin A, Cinque P (2005) Prognostic significance of JC virus DNA levels in cerebrospinal fluid of patients with HIV-associated progressive multifocal leukoencephalopathy. Clin Infect Dis 40:738-744

Brew BJ, Davies NW, Cinque P, Clifford DB, Nath A (2010) Progressive multifocal leukoencephalopathy and other forms of JC virus disease. Nat Rev Neurol 6:667-679

Brickelmaier M, Lugovskoy A, Kartikeyan R, Reviriego-Mendoza MM, Allaire N, Simon K, Frisque RJ, Gorelik L (2009) Identification and characterization of mefloquine efficacy against JC virus in vitro. Antimicrob Agents Chemother 53: 1840-1849

Delbue S, Elia F, Carloni C, Tavazzi E, Marchioni E, Carluccio S, Signorini L, Novati S, Maserati R, Ferrante P (2012) JC virus load in cerebrospinal fluid and transcriptional control region rearrangements may predict the clinical course of progressive multifocal leukoencephalopathy. J Cell Physiol 227: 3511-3517

Ferenczy MW, Marshall LJ, Nelson CD, Atwood WJ, Nath A, Khalili K, Major EO (2012) Molecular biology, epidemiology, and pathogenesis of progressive multifocal leukoencephalopathy, the JC virus-induced demyelinating disease of the human brain. Clin Microbiol Rev 25:471-506

Gasnault J, Costagliola D, Hendel-Chavez H, Dulioust A, Pakianather S, Mazet AA, de Goer de Herve MG, Lancar R, Lascaux AS, Porte L, Delfraissy JF, Taoufik Y, ANRS 125 Trial Team (2011) Improved survival of HIV-1-infected patients with progressive multifocal leukoencephalopathy receiving early 5 -drug combination antiretroviral therapy. PLoS One 6:e20967

Koralnik IJ (2006) Progressive multifocal leukoencephalopathy revisited: has the disease outgrown its name? Ann Neurol 60:162-173

Roche Laboratories Inc. (2003) Lariam (mefloquine) [prescribing information]. Roche Laboratories, Nutley. Available at: http:// www.accessdata.fda.gov/drugsatfda_docs/label/2003/19591slr022 lariam_lbl.pdf. Accessed 18 Apr 2012

Schlagenhauf P, Adamcova M, Regep L, Schaerer MT, Rhein HG (2010) The position of mefloquine as a 21 st century malaria chemoprophylaxis. Malaria J 9:357

Tan CS, Koralnik IJ (2010) Progressive multifocal leukoencephalopathy and other disorders caused by JC virus: clinical features and pathogenesis. Lancet Neurol 9:425-437

Zivadinov R, Rudick RA, De Masi R, Nasuelli D, Ukmar M, PozziMucelli RS, Grop A, Cazzato G, Zorzon M (2001) Effects of IV methylprednisolone on brain atrophy in relapsing-remitting MS. Neurology 57:1239-1247 